\title{
Preserving ovarian function in patients receiving cyclophosphamide
}

\author{
CA Slater ${ }^{1}$, MH Liang ${ }^{1}$, JW McCune ${ }^{2}$, GM Christman $^{3}$ and MR Laufer ${ }^{4}$ \\ ${ }^{1}$ Department of Medicine, Division of Rheumatology, Immunology and Allergy, Harvard Medical School, Brigham and Women's Hospital, Boston, \\ Massachusetts; ${ }^{2}$ Division of Rheumatology, ${ }^{3}$ Division of Obstetrics and Gynecology, University of Michigan Medical Center, University of Michigan \\ Medical School, Ann Arbor, Michigan; and ${ }^{4}$ Division of Gynecology, Department of Surgery, Division of Adolescent/Young Adult Medicine, Department \\ of Medicine, Children's Hospital; Division of Reproductive Endocrinology, Department of Obstetrics and Gynecology, Brigham and Women's Hospital; \\ Department of Obstetrics, Gynecology and Reproductive Biology, Harvard Medical School, Boston, Massachusetts, USA
}

\section{Introduction}

Cyclophosphamide (cytoxan) is an established therapy for the treatment of lupus glomerulonephritis and may well be of value in neurologic and other major organ manifestations unresponsive to standard therapies in systemic lupus erythematosus. Nevertheless, it is potentially toxic with both short- and long-term adverse sequelae. In addition to increased susceptibility to infection, ${ }^{62}$ bone marrow suppression, alopecia, hemorrhagic cystitis and malignancy, women of child-bearing age must weigh the risks of sustained amenorrhea and infertility against the benefit of improved disease control. The risk of infertility from cyclophosphamide is multifactorial and having children is not absolutely contraindicated in lupus patients. In this paper we review the biology of ovarian function, the epidemiology of cyclophosphamide-induced ovarian failure, and the possible strategies for protecting ovarian function in the face of cyclophosphamide therapy.

\section{Mechanism of action and toxicity of cyclophosphamide}

The immunosuppressive actions of cyclophosphamide are complex. Following activation of cyclophosphamide in the liver, multiple metabolites appear in the circulation with varying degrees of immunosuppressive action and toxicity. Although direct toxicity to immunocompetent cells is probably the major me-

*Correspondence: Matthew H. Liang MD, MPH, Division of Rheumatology, Immunology, and Allergy, Brigham and Women's Hospital, 75 Francis Street, Boston, Massachusetts 02115, USA. chanism of immunosuppression, cyclophosphamide is also immunomodulatory in $\mathrm{T}$ cells. The immune effects of cyclophosphamide differ depending on the dose, route of administration, and duration of cyclophosphamide therapy. ${ }^{1-4,54,56}$

As with other alkylating agents in the nitrogen mustard class, rapidly dividing cells are particularly sensitive to the actions of cyclophosphamide. Thus, frequently encountered toxicities include bone marrow suppression and mucosal lining abnormalities. Because cyclophosphamide metabolites are excreted in the urine, hemorrhagic cystitis and bladder cancer are also prominent complications. Urotoxicity can be minimized by limiting the total dose of cyclophosphamide, using bolus rather than daily regimens, intense hydration, and the use of MESNA (sodium 2mercaptoethane sulfonate).

Gonadal failure occurs in both men and women who receive alkylating agents. Since women of reproductive age are the largest group of SLE patients, consideration of preservation of ovarian function is an important issue when reviewing the risks and benefits of using the agent.

\section{Ovarian physiology and cyclophosphamide- induced amenorrhea}

Normal ovarian development begins during the second month of fetal development. Prior to birth, oogonia have already developed to become primary oocytes. At birth, approximately 2 million oocytes are present and no new oogonia are formed. After birth, damaged oocytes are never replaced. Oocytes not incorporated into primordial follicles prenatally undergo atresia. At menarche there are $300000-$ 400000 oocytes and follicular maturation begins. 
Phosphoramide mustard is thought to be responsible for ovarian toxicity, $8,11,31,54,56$ but the exact mechanism is unknown and the mechanism in lupus patients may be different. DNA cross-linking occurs in granulosa cells of experimental animals within $2 \mathrm{~h}$ after injection of cyclophosphamide. An increase in nuclear size is consistent with a G2 cell cycle (when energy required for cell division is stored and when repairs of errors in DNA synthesis occur) phase block. Administration of cyclophosphamide is also associated with a temporary decrease in estradiol levels ${ }^{32}$ consistent with granulosa cell dysfunction. In rats, cyclophosphamide causes a loss of follicular number, with the effect blocked by the concurrent administration of a gonadotropin-releasing hormone agonist (GnRH-a). ${ }^{65}$ Cyclophosphamide has also been shown to have a toxic effect on granulosa cell progesterone production. Damage to granulosa cells might have a secondary toxic effect on oocytes since granulosa cells are regarded as nursing cells for the oocytes through changes in critical intercellular communication of the granulosa cell with the oocyte.

Most of the human data relating to ovarian function after chemotherapy are derived from cancer survivors, particularly young women with a history of Hodgkin's disease or children with Wilms' tumors. Alklylating agents are usually part of a multidrug regimen used to treat these malignancies and thus have not been studied alone. In bone marrow transplant patients, cyclophosphamide is often used alone but at much higher doses $\left(3600 \mathrm{mg} / \mathrm{m}^{2}\right)$ than those used in rheumatic conditions $\left(500-100 \mathrm{mg} / \mathrm{m}^{2}\right)$. After exposure to alkylating agents, human ovaries show fibrosis and follicle destruction with increased levels of follicle-stimulating hormone (FSH) and luteinizing hormone (LH) and decreased estradiol. ${ }^{47,57}$ The result of these changes is hypergonadotropic hypogonadism and subsequent amenorrhea, with likely irreversible ovarian dysfunction and infertility. These hormonal and ovarian side-effects are more common in older patients and those who have received higher cumulative doses of cyclophosphamide. In a study of women treated for breast cancer, the average dose of cyclophosphamide given before the onset of amenorrhea was $20.4 \mathrm{~g}, 9.3 \mathrm{~g}$, and $5.2 \mathrm{~g}$ for women in their twenties, thirties and forties, respectively. ${ }^{63}$ Younger patients tend to tolerate higher doses of alkylating agents. ${ }^{47,55}$ Menstrual patterns prior to exposure to cyclophosphamide do not appear to be independent risk factors for early menopause. ${ }^{5,9}$ When lupus nephritis patients were studied retrospectively, persistent amenorrhea was dependent on route of administration, age of patient, cumulative dose and duration of therapy, with age greater than 30 years and large cumulative doses $(>300 \mathrm{mg} / \mathrm{kg}$ ) being worse prognostic indicators. ${ }^{5,6,9,10,48,58}$ In one retrospective study looking at monthly intravenous bolus cyclophosphamide, $12 \%$ of patients under 25 years of age, $27 \%$ of patients aged $26-30$ years and $62 \%$ of patients aged 31 years or over experienced sustained amenorrhea for at least 12 months after the cessation of the cyclophosphamide. ${ }^{9}$ The published data do not permit a clear picture of whether there is a threshold cumulative dose associated with amenorrhea. One study of 92 female lupus nephritis patients taking daily oral cyclophosphamide at a dose of $1-$ $2 \mathrm{mg} / \mathrm{kg}$ /day found that the $27 \%$ of patients who developed sustained amenorrhea received a mean cumulative dose of $32.6 \mathrm{~g}$ of cyclophosphamide versus $22.4 \mathrm{~g}$ in the group that continued menstruating. 5

It is not clear whether the length of amenorrhea after cylophosphamide treatment predicts recovery of menses or fertility, nor do we know whether recovery of menses protects a given patient from future early menopause.

\section{Preservation of ovarian function}

Preservation of ovarian function in women receiving cyclophosphamide was first studied in the early 1980 s in patients with Hodgkin's disease. ${ }^{47}$ One study employed combination oral contraceptive pills (OCPs) to suppress ovarian function during chemotherapy ${ }^{46}$ but included only 6 patients. Three of the six women underwent ovarian biopsy after chemotherapy and had the same number of follicles compared with pretreatment biopsies. One woman went on to become pregnant. On this basis, the authors suggest that OCPs may have a protective effect on fertility in these patients. There are no controlled studies to date addressing the potential protective role of OCPs.

\section{Gonadotropin-releasing hormone physiology}

Gonadotropin-releasing hormone (GnRH) acts to suppress ovarian function, creating a quiescent or 'prepubertal' state, and thus could theoretically protect ovarian function during cytotoxic therapy. $\mathrm{GnRH}$ is a decapeptide with a biological half-life of 2-8 min that is produced in the arcuate nucleus of the hypothalamus and secreted in a pulsatile manner. It induces synthesis and release of the pituitary gonadotroph follicle-stimulating hormone (FSH) and luteinizing hormone. When GnRH is given by continuous 
intravenous infusion, increased levels of FSH and $\mathrm{LH}$ are seen in the first $4 \mathrm{~h}$ followed by progressive and sustained decrease in these hormones, resulting in 'medical castration'. 13,23 There are two proposed mechanisms for the continuous action of GnRH. One is a desensitization with uncoupling of the activated GnRH receptor from its binding complex. The other is a down-regulation of the number of available receptors for binding.

\section{Synthetic GnRH agonists}

Various synthetic GnRH agonists (GnRH-a) are available that are more potent than the natural hormone (Table 1). The analogs mimic the action of continuous high-dose GnRH. Females receiving synthetic GnRH-a have serum estradiol concentrations similar to those levels seen naturally in postmenopausal women. ${ }^{23}$ There are four preparations approved for use in the United States, but more are available abroad (Table 1). Leuprolide acetate (Lupron) is available in subcutaneous or depot formulations (1 month and 3 month) and is approved for the treatment of prostate cancer, endometriosis, leiomyomata, ${ }^{49}$ dysfunctional uterine bleeding, in vitro fertilization and precocious puberty. ${ }^{23,38,40,41}$ The other available agonists are nafarelin acetate (Synarel), an intranasal spray preparation, goserelin (Zoladex), a sustainedrelease formulation (lasting 1 or 3 months), and Histrelin, which is used for precocious puberty but is not available in the United States. The use of GnRH-a is increasingly used to reversibly suppress endogeneous ovarian production of estradiol and progesterone.

Most women who receive a GnRH-a develop amenorrhea with decreased hormone levels within 3-8 weeks. ${ }^{38}$ Menses usually returns approximately 6
Ovarian function and cyclophosphamide

CA Slater et al

weeks after the last subcutaneous injection or 10 weeks after a depot monthly injection. For women whose menses do not recur in this time frame, evaluation of serum hormone levels will help evaluate whether the amenorrhea is a result of anovulation secondary to chronic disease (serum FSH under $10 \mathrm{mIU} / \mathrm{ml}$ on day 3 of menses) or premature ovarian failure $(\mathrm{FSH}>40 \mathrm{mIU} / \mathrm{ml})$.

\section{GnRH-a side-effects}

More than $75 \%$ of patients develop hot flashes ${ }^{18,19,23,24}$ when exposed to GnRH-a, which tend to decrease in intensity and frequency after the first few injections. Loss of bone density because of relative estrogen deficiency is probably the most important side-effect for rheumatology patients given the frequency of concurrent and long-term corticosteroid utilization. Other significant side-effects include irregular vaginal bleeding, depression, headache and insomnia. Most of the current data on side-effects come from the treatment of endometriosis, dysfunctional uterine bleeding and leiomyomata reported by the Leuprolide Study Group. ${ }^{17,19}$ Unfortunately, the bone mineral density data were collected after the initial data collection and what is reported is pooled from different sites and substudies. ${ }^{17,21}$ Thus there was no standardization of measurement of bone density and any correlation between bone mineral density and estrogen levels was not addressed. Finally, the available data do not address potential confounders that affect the risk of developing osteoporosis, such as tobacco use, weight, exercise, caffeine, prednisone and other medications, or chronic disease, Some studies do control for calcium supplementation. Sidenius and colleagues studied the effects of intranasal nafarelin on bone metabolism in women

Table 1 GnRH agonists

\begin{tabular}{|c|c|c|c|c|}
\hline Generic name (brand name) & Relative potency ${ }^{\mathrm{a}}$ & Half-life in humans (min) & Indications & Modes of adminstration \\
\hline Tryptorelin (Decapeptyl) & 100 & 50 & CPP, Endo, PC & $\mathrm{SC}, \mathrm{IM}$ \\
\hline Buserelin (Superfact) & $?$ & 75 & PC, Endo & SC induction followed by IN \\
\hline Histrelin & 100 & $<60$ & $\mathrm{CPP}^{\mathrm{b}}$ & $\mathrm{SC}$ \\
\hline Leuprorelin (Lupron) & $15^{\mathrm{c}}$ & 180 & $\begin{array}{c}\text { CPP, } \mathrm{PC}^{\mathrm{b}} \\
\text { Endo }^{\mathrm{b}}\end{array}$ & SC,IM \\
\hline Nafarelin (Synarel) & $200-300$ & 240 & Endo $^{\mathrm{b}}$ & IN \\
\hline Goserelin (Zoladex) ND & ND & $7 \mathrm{hr}$ & $\mathrm{PC}^{\mathrm{b}}$, Endo $^{\mathrm{b}}$ & Implant \\
\hline
\end{tabular}

Adapted from ref. 14.

a Potency calculated on basis of estrus suppression test in rats; native GnRH is 1.

${ }^{\mathrm{b}}$ Approved for clinical use in United States.

${ }^{\mathrm{c}}$ Test of circulating LH increase in male rates; in LH augmentation test in rats, relative potency was 100. Abbreviations:

CPP, central precocious puberty; Endo, endometriosis; PC, prostate cancer; SC, subcutaneous; IN, intranasal; IM, intramuscular. 
with laparoscopic proven endometriosis. ${ }^{22}$ All subjects in whom measurements were obtained had a bone mineral density (BMD) decrease of $2-6 \%$ after 6 months of therapy as measured by single-photon absorptiometry (SPA), dual-photon absorptiometry (DPA) and urinary calcium:creatinine ratio or hydroxyproline: creatinine ratio. All bone density measurements returned to baseline 6 months after discontinuing the nafarelin. ${ }^{22}$ In a randomized, double-blind multicenter trial of depot Lupron (leuprolide acetate) for endometriosis, spine BMD decreased $3.6-11.8 \%$ with different methods of measurement between centers. ${ }^{17}$ In one study, 6 of 8 patients had complete recovery of BMD loss 6 months after Lupron therapy was stopped; other authors report only partial recovery of lost bone density if the GnRH-a is stopped after 6 months of therapy. ${ }^{17,20,21,44}$ Fogelman reports a $4.5 \%$ decrease in BMD of the spine and 3\% in the femur as measured by dual X-ray absorptiometry (DXA) in a placebo-controlled double-blind study of 60 women treated with Zoladex (goserelin acetate) for premenstrual syndrome. ${ }^{45}$ Cann summarized data from multiple centers where $\mathrm{GnRH}$ agonists were used to treat endometriosis and leiomyomata and found that most studies showed some significant BMD loss. ${ }^{59}$ Some recovery of lost bone density is reported if the GnRH-a is stopped after 6 months of therapy. ${ }^{20,21,44}$ Damario found persistent decreases in BMD up to 48 weeks after treatment of endometriosis with Zoladex. ${ }^{39}$ Many studies, however, have no follow-up with respect to recovery of BMD. ${ }^{17}$

Overall, the clinical impression is that Lupron alters bone metabolism through its effect on estrogen secretion although a GnRH-a-induced inhibition of growth hormone, and insulin-like growth factor-I may also contribute to alterations in the calcium metabolism in bones. The magnitude of change depends on the skeletal site and measurement techniques, and the effect on bone is probably reversible. Lupron is generally not given for longer than 6 months and its effects on bone mineral density for longer than 6 months is not known. Since some treatment protocols for lupus nephritis frequently use cyclophosphamide for 24 36 months or longer, these patients might be exposed to Lupron for longer periods of time if given to protect gonadal function. The issue of lost bone mineral density is of paramount importance for lupus patients since they are frequently on high doses of steroids for prolonged periods, ${ }^{1-4,9}$ a known and powerful risk factor for osteoporosis. Finally, there are no large studies of fertility and fecundity in lupus patients co-treated with cyclophosphamide and GnRH-a.

\section{GnRH-a for preservation of ovarian function}

The mechanism of action of GnRH agonists in protecting ovarian function in humans is unknown. Some possible mechanisms include a centrally mediated suppression of gonadotropins, direct suppression of gonadotropin receptors in the gonad, and a reduction of biologic activity of gonadotropins.

In rat models of cyclophosphamide-induced ovarian failure, GnRH-a seem to decrease the number of follicles undergoing further development at the time of exposure to cyclophosphamide, thus rendering them less susceptible to the toxic effects of alkylating agents. $^{12,14,32}$ Montz and colleagues evaluated rats given cyclophosphamide with concurrent Lupron or progesterone, and demonstrated a protective effect of the Lupron on fertility, but not on fecundity as litter sizes were significantly smaller than control litters. ${ }^{7}$ Ataya and colleages evaluated monthly Lupron injections in rhesus monkeys as a possible means for inhibiting cyclophosphamide-induced ovarian failure and found that Lupron therapy protected the ovaries against accelerated follicular depletion. In this study small follicles were most severely affected. ${ }^{15,16}$ Similarly, in humans, ovarian tissue taken from women who received alkylating agents has a characteristic depletion of primordial follicles. ${ }^{46}$

A recent study of young women receiving chemotherapy for treatment of lymphoma showed a significant protective effect against irreversible ovarian failure from the co-treatment with a GnRH agonst. ${ }^{66}$ The authors found that of those treated with the GnRH-a, 94\% resumed spontaneous ovulation and menses within 3-8 months of termination of the combined chemotherapy/GnRH-a co-treatment. Of those who were treated without the GnHR-a, $61 \%$ experienced premature ovarian failure.

\section{Preservation of bone mineral density}

If a GnRH agonist is to be used to protect ovarian function, then prevention of bone loss must also be addressed. If maximal suppression of gonadal function is necessary, then the addition of estrogen and/or progesterone similar to a postmenstrual hormone replacement therapy (HRT) or 'add-back' could be considered. In a placebo-controlled trial, Leather and colleagues showed that adding back estradiol valerate $(2 \mathrm{mg} / \mathrm{d})$ with norethesterone $(5 \mathrm{mg}$ on days 22-28) to women receiving Zoladex for 6 months for PMS prevented the loss of BMD seen in patients not receiving add-back therapy. ${ }^{37}$ However, only $10 / 19$ women in the add-back treatment group 
completed the trial. There is controversy, however, regarding the safety of hormone replacement therapy in SLE. ${ }^{34-36}$ Estrogen is implicated in the induction and exacerbation of SLE. ${ }^{35,60,61}$ Petri and Robinson reviewed the use of OCPs in SLE and conclude that OCPs should probably be avoided in women with active renal disease because of potential increased risk of flare. Furthermore, OCPs may place SLE patients with anti-phospholipid antibodies at increased risk for thrombosis. ${ }^{34}$ These risks, however, may be diminished with other estrogen preparations such as conjugated estrogen or through the use of transdermal estradiol delivery systems. Thus, these risks must be evaluated against the potential benefit of preserving BMD, contraception and possible preservation of ovarian function. Non-hormonal therapy to prevent bone loss is aimed at blocking bone resorption with bisphosphonates or by inhibiting osteoclast activity with calcitonin. Bisphosphonates inhibit bone resorption and reduce bone turnover. Etidronate and aldendronate (Fosamax) both increase BMD in postmeopausal and steroid-induced osteoporotic patients, and alendronate reduces risk of fractures. ${ }^{33}$ Supportive measures for bone formation such as calcium supplementation, weight-bearing exercise ${ }^{18}$ and limitation of alcohol and tobacco use may help retard bone loss.

\section{Preservation of ovarian function}

Based on the preliminary data in the literature, it is reasonable to offer women of reproductive age the option to utilize GnRH-a co-treatment in an attempt to preserve ovarian function. At the Brigham and Women's Hospital, our recommendation for women interested in this co-treatment is Depot-Lupron 3month formulation $11.25 \mathrm{mg}$ for two injections (total 6 months of therapy) started 3-4 weeks prior to the initiation of cyclophosphamide, and then 'pulse' Lupron with $7.5 \mathrm{mg}$ of the monthly formulation one month prior to and on the day of the 'pulse' cyclophosphamide. The first Lupron injection may by necessity be given closer to the first cyclophosphamide dose if the clinical situation is dire. Pretreatment BMD measurement is recommended, and repeated if the GnRH-a is to be continued for longer than 6 months. If a patient's bone density is below normal or the patient is on steroids, bisphosphonate therapy may be offered, but concerns exist about effects on future fetuses from bone treated with biphosphonates. All patients are supplemented with calcium and vitamin D. The use of azathioprine following monthly pulse cyclophosphamide can be considered in individual cases to minimize cyclophosphamide and GnRH-a exposure.

At the University of Michigan, informed consent as a research study is obtained. Patients receive $3.75 \mathrm{mg}$ of Lupron acetate at least 10 days before the next injection of cyclophosphamide. When this cannot be done before the first pulse, Lupron is started between the first and second pulses and Lupron is given for 6 months when consideration is given to changing cyclophosphamide to azathioprine. Patients routinely receive estrogen supplementation after 1-2 months, with estroderm patch either 0.05 or $0.1 \mathrm{mg}$ every $3 \frac{1}{2}$ days. The lower strength is used first and, if symptoms continue the higher dose patch is used. This is generally continued for at least a year.

\section{Other options for preserving reproductive function}

The main alternative to GnRH-a analogs for preserving ovarian function is cryopreservation of the oocytes. Historically there have been problems with freezing of oocytes. The microtubule spindle of the mature oocyte is sensitive to temperature changes, resulting in nondisjunction of chromosomes. Cryoprotectants used in the freezing process harden the zona pellucida, interfering with fertilization. ${ }^{26}$ Early preliminary studies suggest up to $51 \%$ survival of thawed rodent oocytes, but murine tissue was more promising. ${ }^{27}$ Methods of optimal freeze/thaw technique and the use of alternative cryoprotectants are being studied for both ovarian tissue and oocyte cryopreservation. ${ }^{25,28,29,30,43,49,50,51}$ Persistent hurdles include viability of the thawed tissue and chromosal abnormalities once the thawed oocytes mature, ${ }^{49}$ but evolving improvements, including cryopreservation of primordial follicles, are making this option more feasible. A few cases of successful pregnancies from cryopreserved ooctyes have been reported. ${ }^{64}$

Surgical autografting of ovarian tissue is a theoretical option, but there are controversial animal data on whether it is effective. ${ }^{42,51,52}$ In this technique, ovarian tissue is frozen prior to chemotherapy and then regrafted after exposure. Technical problems include graft ischemia and marked reduction of primordial follicles, ${ }^{53}$ thus limiting the practicality of this option at present. No successful pregnancies have thus far been reported.

Embryo cryopreservation and future cryopreservation of oocytes, as well as surrogate gestational carriers of fertilized embryos, are exciting options to offer patients prior to the initiation of cytotoxic therapy. These techniques may necessitate postponing 
chemotherapy for approximately 1 month and thus may be impractical based on the urgency of the clinical situation.

\section{Recommendation for clinical practice}

In conclusion, there are numerous issues that warrant family planning and counselling of female patients about to undergo cyclophosphamide therapy for lupus nephritis. There is no simple algorithm to offer, nor is there universal agreement even among the experts about the optimal ways of preserving fertility, and complex patients may require the guidance of other specialists to make informed decisions. We offer general guidelines while recognizing that other approaches are also reasonable for some of the more common scenarios that may be encountered.

Before a thoughtful discussion about family planning can take place, it is probably wise for young female patients/couples to undergo evaluation with an experienced obstetrician/maternal fetal specialist to address whether pregnancy would be safe for a given patient. There are many considerations, including prior obstetrical history, hypertension, anti-phospholipid antibodies, co-morbid illnesses such as renal failure, pulmonary hypertension, prior thromboembolic disease, cardiomyopathy or other cardiac pathology and SLE activity, to name a few. A consultation with a gynecologist with a specialty in reproductive medicine may be helpful. It would also be wise to address male fertility issues as well at an initial family planning visit in order to more fully investigate the couple's possibility of pregnancy. If a woman is not interested in ever having children or plans on adopting in the future, then informed consent and counselling for risk of amenorrhea and possible early menopause and the other adverse effects of cyclophosphamide is sufficient. Many lupus patients are young, however, and may change their view with regard to wanting children.

For women under 16 years, a GnRH-a protocol is not advised because its effects on growing bone are unknown. After careful consideration of all the risk and benefits, these patients are often offered low-dose combination OCPs to suppress ovarian function. It should be remembered that OCPs should be avoided in SLE patients with anti-phospholipid antibodies ${ }^{34}$ or who are otherwise at high risk for thromboembolic disease.

For a younger woman who has not yet had children but has a male partner, and in whom pregnancy is not otherwise contraindicated, there are several options. This group is at lower risk for infertility than older patients, but again the anticipated cumulative cyclophos- phamide dose appears to play a large role. The most aggressive approach, if the time of initiation of the chemotherapy permits, would be to consider assisted reproductive technology and cryopreservation of embryos before exposure to cyclophosphamide and then attempts at hormonal suppression as well. If these techniques are not elected, then a GnRH-a protocol should be seriously considered. There are new experimental protocols for freezing oocytes that attempt to address the technical limitations of viability and chromosomal abnormalities. These may be available to patients who do not have a partner but would like to retain reproductive function for the future. With all strategies, success is unpredictable and patients may choose no intervention and take their chances on recovering ovarian function. We have no sure way of predicting the likelihood of permanent ovarian failure and it is even more difficult to predict whether the patient will need more cyclophosphamide.

Women over the age of 30 years stand the greatest chance of infertility and premature menopause. If a woman in this category clearly wants to have children in the future, she should consider some precautionary measures. One such option might be to consider a cryopreservation protocol if time permits. It should be remembered that for women who have undergone premature ovarian failure, hormonal manipulation with exogeneous estrogens and progesterones and embryonic transfer techniques can make carrying a pregnancy possible. Gestational surrogate carriers are legal in some areas and may offer the chance for gestation of a frozen embryo if the mother is unable to carry a pregnancy. Evaluation for a $\mathrm{GnRH}-\mathrm{a}$ protocol is recommended in this age group.

In summary, there is need for more research on cyclophosphamide-induced ovarian failure, improved prediction rules for its occurrence, and evaluation of the risks and benefits of strategies using GnRH-a to preserve ovarian function. In the mean time, pregnancy is not necessarily contraindicated in lupus patients $^{34,36,48,53}$ and thus frank discussion of what is available as well as the available strategies to attempt preservation of ovarian function is an affirmation of how important these issues are in a woman's health care and her quality of life.

\section{Acknowledgements}

Cindi A. Slater was supported by NIH training grant ARO 7530-10. Matthew H. Liang has been supported by NIH grants AR 36308, AI 31599, and the Arthritis Foundation Clinical Science Grant. 


\section{References}

1 Steinberg AD, Steinberg SC. Long term preservation of renal function in patients with lupus nephritis receiving treatment that includes cyclophosphamide versus those treated with prednisolone alone. Arthritis Rheum 1991; 34 (8): 945-950.

2 Balow JW, et al. Lupus nephritis. Ann Intern Med 1987; 106: 79-94.

3 Boumpas DT, et al. Controlled trial of pulse methyl prednisolone versus two regimens of pulse cyclophosphamide in severe lupus nephritis. Lancet 1992; 340: 741-745.

4 Levey AS, et al.. The Lupus Nephritis Collaborative Study Group. Progression and remission of renal disease in the lupus nephritis collaborative study. Ann Intern Med 1992; 116: 114-123.

5 Wang CL, Wang F, Bosco JJ. Ovarian failure in oral cyclophosphamide treatment for systemic lupus erythematosus. Lupus 1995; 4: 11-14.

6 Belmont HM, Storch M, Buyon J. Abramson S. New York University/Hospital for Joint Disease experience with intravenous cyclophosphamide treatment: efficacy in steroid unresponsive lupus nephritis. Lupus 1995; 4: 104-108.

7 Montz FJ, Wolff AJ, Gambone, JC. Gonadal protection and fecundity rates in cyclophosphamide-treated rats. Cancer Res 1991; 51: 2124-2126.

8 Klippel JH. Cyclophosphamide: ovarian and other toxicities. Lupus 1995; 4: 1-2.

9 Boumpas DT et al. Risk for sustained amenorrhea in patients with systemic lupus erythematosus receiving intermittent pulse cyclophosphamide therapy. Ann Intern Med 1993; 119: 366-369.

10 Langevitz P, Klein L, Pras M. Many A. The effect of cyclophosphamide pulses on fertility in patients with lupus nephritis. Am J Reprod Immunol 1992; 28: 157-580.

11 Roubenoff R. Effects of antiinflammatory and immunosuppressive drugs on pregnancy and fertility. Semin Arthritis Rheum 1988; 18 (2): 88-110.

12 Karashima T, Zalatni A, Schally AV. Protective effects of analogs of luteinizing hormone-releasing hormone against chemotherapy-induced testicular damage in rats. Proc Natl Acad Sci USA 1988; 85: 2329-2333.

13 Tummon IS, et al. A randomized, prospective comparison of endocrine changes induced with intranasal leuprolide or danazol for treatment of endometriosis. Fertil Steril 1989; 51: 390-394.

14 Ataya $\mathrm{KM}$ et al. A luteinizing hormone-relating agonist for the prevention of chemotherapy-induced ovarian follicular loss in rats. Cancer Res 1985; 45: 2651-2656.

15 Ataya KM, Rao LV, Lawrence E, Kimmel R. Luteinizing hormonereleasing hormone agonist inhibits cyclophosphamide induced ovarian follicular depletion in rhesus monkeys. Biol Reprod 1995; 52: 365-372.

16 Ataya KM, Pydyn E, Ramahi-Ataya, Orton CG. Is radiation induced ovarian failure in rhesus monkeys preventable by luteinizing hormonereleasing hormone agonists? J Clin Endocrinol Metals 1995; 80 (3): 790-794.

17 Dlugi AM, Miller JD, Knittle J, Lupron depot (leuprolide acetate for depot suspension) in the treatment of endometriosis: a randomized, placebo-controlled, double-blind study. Fertil Steril 1990; 54: 419-426.

18 Lemay A, Surrey ES, Friedman AJ. Extending the use of gonadotropinreleasing hormone agonists. The emerging role of steroid and nonsteroid agents. Fertil Steril 1994; 61 (1): 21-34.

19 Friedman AJ, et al. for the Leuprolide Study Group. Treatment of leiomyomata uteri with leuprolide acetate depot: a double-blind, placebo-controlled, multi center study. Obstet Gynecol 1991; 77: 720-725.

20 Fogelman, I. Gonadotropin-releasing hormone agonists and the skeleton. Fertil Steril 1992; 57: 715-724.

21 Scialli AR, Jestila KJ, Simon JA. Leuprolide acetate and bone mineral density measured by quantitive radiography. Fertil Steril 1993; 59 (3): 674-676

22 Johansen JS, et al. The effect of a gonadotropin-releasing hormone agonist analog (nafarelin) on bone metabolism. J. Clin. Endocrinol Metab 1988; 67: 701-706.
23 Barbieri RL, Friedman AJ. Gonadotropin Releasing Hormone Analogs. New York: Elsevier Science, 51, 1991.

24 Plosker GL, Brogden RN. Leuprorelin. Drugs 1994; 48 (6): 930-967.

25 Tobback C, Hugh S, Foote RH. A procedure for cyropreservation of hamster oocytes yielding highly conserved oocytes suitable for sperm penetration tests. Fertil Steril 1991; 55 (1): 184-188.

26 Hammitt DG. Conditions of oocyte storage and use of noninseminated as compared with inseminated non fertilized oocytes for the hemizona assay. Fertil Steril 1993; 60: 131-163.

27 Harp R et al. Cryopreservation of murine ovarian tissue. Cryobiol 1994; 31: 336-343.

28 Gook DA, Osborn SM, Bourne H, Johnson WIH. Fertilization of human oocytes following cyropreservation: normal karyotypes and absence of stray chromosomes. Hum Reprod 1994; 9 (4): 684-691.

29 Hunter JL. Cryopreservation of human prophase I oocytes collected from unstimulated follicles. Fertil Steril 1994; 61 (6): 1077-1082.

30 Toth TL et al. Cryopreservation of human prophase I oocytes collected from unstimulated follicles. Fertilization and in vitro development of cryopreserved human prophase I oocytes. Fertil Steril 1994; 61 (5): 891-894.

31 Gutmann JN. Cancer and infertility. Infertil Reprod Med Clin North Am 1996; 7 (3): 935-952.

32 Ataya KM, Valeriote FA, Ramahi-Ataya AJ. Effect of cyclophosphamide on the immature rat ovary. Cancer Res 1989; 49: 1660-1664.

33 Hochberg MC. Bisphosphonates in the treatment of osteoporosis. $J$ Clin Rheumatol 1997; 3: 537-539.

34 Petri M, Robinson C. Oral contraceptives and systemic lupus erythematosus. Arthritis Rheum 1997; 40 (5): 797-803.

35 Arden NK, Lloyd ME, Spector TD, Hughes GRV. Safety of hormone replacement therapy (HRT) in systemic lupus erythematosus (SLE). Lupus 1994; 3: 11-13.

36 Buyon JP et al Can women with systemic lupus erythematousus safely use exogenous estrogens? J Clin Rheumatol 1995; 1 (4): 205-212.

37 Leather AT, Studd JWW, Watson NR, Holland EFN. The prevention of bone loss in young women treated with GNRHa analogues with 'add back' estrogen therapy. Obstet Gynecol 1993; 81 (1): 104-107.

38 Laufer MR, et al. Inducing amenorrhea during bone marrow transplantation. J Reprod Med 1997; 42 (9): 537-541.

39 Damario MA, Rock JA. Gosrelin (Zoladex) versus danazol for endometriosis: the North American experience. B J Obstet Gyanecol 1994; 101 (S10): S13-S18.

40 Laufer MR, Rein MS. Treatment of abnormal uterine bleeding with gonadotropin-releasing hormone analogues. Clin Obstet Gynecol 1993; 36 (3): 668-678.

41 Henzl MR. Gonadotropin-releasing hormone and its analogues: from laboratory to bedside. Clin Obstet Gynecol 1993; 36 (3): 617-635.

42 Newton $\mathrm{H}$ et al. Low temperature storage and grafting of human ovarian tissue. Hum Reprod 1996; 11 (7): 1487-1491.

43 Zhang $\mathrm{J}$ et al. Extracorporeal development and ultrarapid freezing of human fetal ova. J Assist Reprod Gent 1995; 12 (6): $361-368$.

44 Dawood MY, Ramos J, Khan-Dawood FS. Depot Leuprolide acetate versus danazol for treatment of pelvic endometriosis: changes in vertebral bone mass and serum estradiol and calcitonin. Fertil Steril 1995; 63 (6): 1177-1183.

45 Fogelman I, et al. Goserelin (Zoladex) and the skeleton. Br J Obstet Gynaecol 1994; 101 (S10): S19-S23.

46 Chapman RM, Sutcliffe SB. Protection of ovarian function by oral contraceptives in women receiving chemotherapy for Hodgkins disease. Blood 1981; 58 (4): 849-851.

47 Gradishar WJ, Schilsky RL. Ovarian function following radiation and chemotherapy for cancer. Semin Oncol 1989; 16 (5): 425-436.

48 Bermas BL, Hill JA. Effects of immunosuppressive drugs during pregnancy. Arthritis Rheum 1995; 38 (12): 1722-1732.

49 Hovatta $\mathrm{O}$, et al. Cryopreservation of human ovarian tissue using dimethylsulphoxide and propanediol-sucrose as cryoprotectants. Hum Reprod 1996; 11 (6): 1268-1272.

50 Candy CJ, Wood MJ Whittingham DG. Follicular development in cryopreserved marmoset ovarian tissue after transplantation. Hum Reprod 1995; 10 (9): 2334-2338.

51 Cox SL, Shaw J, Jenkin G. Transplantation of cryopreserved fetal ovarian tissue to adult recipient in mice. J Reprod Fertil 196; 107: 315 322 
52 Gosden RG, Baird DT, Wade JC, Webb R. Restoration of fertility to oophorectomized sheep by ovarian autografts stored at $-196^{\circ} \mathrm{C}$. Human Reprod 1994; 9 (4): 597-603.

53 Ramsey-Goldman R, et al. Pregnancy outcome in women with systemic lupus erythematosus treated with immunosuppressive drugs. J. Rheumatol 1993; 20 (4): 1152-1157.

54 Kovarsky J. Clinical pharmacology and toxicology of cyclophosphamide: emphasis on use in rheumatic diseases. Semin Arthritis Rheum 1983; 12 (4): 359-371.

55 Sanders JE et al. Late effects on gonadal function of cyclophosphamide, total-body irradiation, and marrow transplantation. Transplantation 1983; 36 (3): 252-255.

56 Clements PJ, Davis, J. Cytotoxic drugs: their clinical application to the rheumatic diseases. Semin. Arthritis Rheum 1986; 15 (4): 231-254.

57 Warne GL, Fairley KF, Horbs JB, Martin FIR. Cyclophosphamideinduced ovarian failure. $N$ Eng $J$ Med 1973; 289: 1159-1162.

58 Rivkees SA, Crawford JD. The relationship of gonadal activity and chemotherapy-induced gonadal damage. JAMA 1988; 259: 2123-2125.

59 Cann CE et al. Reversible bone loss is produced by the GNRHa agonist nafarelin. In: Cohn DV, Martin TJ, Meunier A (eds). Calcium Regulation and Bone Metabolism. Amsterdam: Elsevier Science, 1987, 123-127.
60 Lahita RG, Bucala R, Bralow HL, Fishman J. Determination of 16 alpha-hydroxyesterone by radioimmunoassay in systemic lupus erythematosus. Arthritis Rheum 1985; 18: 1122-1127.

61 Sanchez-Guerrero J, et al. Past use of oral contraceptives and the risk of developing systemic lupus erythematosus. Arthritis Rheum 1997; 40 (5): 804-808.

62 Martin F, et al. Side effects of intravenous cyclophosphamide pulse therapy. Lupus 1997; 6: 254-257.

63 Koyama $\mathrm{H}$, et al. Cyclophosphamide-induced ovarian failure and its therapeutic significance in patients with breast cancer. Cancer 1977; 39: 1403-1409.

64 Polak de Fried E, et al. Pregnancy after human donor oocyte cryopreservation and thaw in association with intracytoplasmic sperm injection in a patient with ovarian failure. Fertil Steril 1998; 69 (3): $555-557$.

65 Ataya KM et al. Inhibition of rat ovarian $\left[{ }^{3} \mathrm{H}\right]$ thymidine uptake by luteinizing hormone releasing hormone agonists: a possible mechanism for preventing damage by cytotoxic agents. Cancer Res 1988; 48 $7252-7256$.

66 Blumenfeld Z, et al. Prevention of irreversible chemotherapy-induced ovarian damage in young women with lymphoma by a gonadotropinreleasing hormone agonist in parallel to chemotherapy. Hum Reprod 1996; 11: $1620-1626$. 